\title{
Spatial Distribution and Demoraphic Characteristic of Gujjars in Jammu Division: A Case Study of Rajouri and Poonch Districts
}

\author{
Nawaz Ahmed
}

\begin{abstract}
The spatial distribution of ethnic groups and tribal population is an interesting area in which the social and cultural geographers and sociologists are increasingly probing. For the ethnic minorities, especially for the transhumant and migratory people land and forest are the source of life. The present paper is an attempt to examine the spatial distribution and demographic characteristic of Gujjars and Bakarwals with special focus on their population and settlement in Rajouri and Poonch districts of Jammu division of Jammu and Kashmir State of India. To identify the Gujjars settlements in the Rajouri and Poonch district. The socio and economic backwardness of the Gujjars and Bakarwals is a function of rugged topography, hilly terrain, prejudices, orthodoxy, illiteracy and the traditional mode of life. There is a conspicuous shift from transhumance to agricultural and non-agricultural sectors among the Gujjars and Bakarwals.
\end{abstract}

Key Words: Tribal Population, Gujjars, Bakarwals, Backwardness, rugged topography, hilly terrain, prejudices, orthodoxy.

The spatial distribution of ethnic groups and tribal population is an interesting area in which the social and cultural geographers and sociologists are increasingly probing. For the ethnic minorities, especially for the transhumant and migratory people land and forest are the source of life. Although transhumant tribal people vary widely in their customs, tradition, culture and impact on the land, all consider the earth like a parent and revere it accordingly. In their opinion, 'Mother earth' is the centre of the universe, the core of their culture, the origin of their identity as a people. The land connects them with their past (as the home of the ancestors), and with the future. In this way ethnic group's indigenousness carries with it a sense of belonging to a place. In their opinion land cannot be given or taken away. We belong to the land. Our spirituality, our culture and our social life depends upon it. At the heart of this deep bound is a perception, an awareness, that all of life -mountains, rocks, rivers, animals, plants, insects, people are inseparably interconnected. In their society, material and spiritual words are woven together in one complex web, all living things imbued with a sacred meaning.

According to Hayden Burgers "the earth is the foundation of tribal and indigenous peoples; it is the seat of the spirituality, the foundation from which our culture and language flourish. The earth is our historian, the keeper of events and the bones of our forefathers. Earth provides us with food, cloth, shelter and medicine. It is the source of our independence, it is our mother, we don't dominate her, and we must harmonize with her.

In the developed world, land is in the hands of private individuals, corporate investors, or the state and can be disposed of all the will of the owner. For tribal people like the Gujjars and Bakarwals of the Jammu and Kashmir state land, especially pastures are held collectively for the community in which the families have customary and traditional rights to graze their cattle, goats and sheep. According to indigenous law, man kind can never be more than a trustee of land, with a collective responsibility to preserve it. These people, still in harmony with the environment around them, don't isolate themselves from other livings, nor consider one creature superior to another their philosophy of life is 'from every one according to his capacity and to every one according to his needs'.

The present paper is an attempt to examine the spatial distribution and demographic characteristic of Gujjars and Bakarwals with special focus on their population and settlement in Rajouri and Poonch districts of Jammu division of Jammu and Kashmir State of India. The main objectives of the present paper are as under:

To identify the Gujjars settlements in the Rajouri and Poonch district, the geo-climatic and socio-economic factors responsible for the spatial distribution of Gujjars settlements in the region.

To prepare an initiary of the resource base (land, pasture, forest, water and minerals) of the Rajouri and Poonch districts and discuss the transhumant patterns of the Gujjars and Bakarwals,

To analyses the characteristic of the Gujjars population and the main hurdles responsible for socio-economic transformation of the Gujjars and Bakarwals.

To suggest a suitable strategy for the social wellbeing, economic prosperity of the Gujjars and Bakarwals of the region to make the resources sustainable and to enhance the resilience characteristic of the physical endowments.

The socio and economic backwardness of the Gujjars and Bakarwals is a function of rugged topography, hilly terrain, prejudices, orthodoxy, illiteracy and the traditional mode of life. The Sedentrisation of 
Gujjars and Bakarwals in the Siwalik and the side valleys of the lesser Himalayas have accelerated the processes of socio-economic transformation of the transhumant of the region. There is an increasing trend to give up the traditional practice of transhumant and to engage them in the business, service and territory sector.

The division of Jammu Rajouri and Poonch districts are located lies between $32^{\circ} 50^{\prime} \mathrm{N}$ to $33^{\circ} 30^{\prime}$ and $74^{\circ} 24^{\prime}$ E to $75^{\circ} 18^{\prime} \mathrm{E}$ longitudes. The total population of Jammu division (2001) was 4395712 out of which 298845 were Gujjars and Bakarwals constitute a significant proportion of the total population of the Jammu division.

The state of Jammu and Kashmir is characterized with great ethnic, linguistic and religious diversity. It is inhabited by various ethnic groups, castes, sub castes and religious minorities. Each of this group has common ancestory, cultural traditions, varying in their gamut of life with a strong feeling of belonging cohesiveness, living as a minority in a wider society. Each ethnic group and community is a repository of distinctive cultural traditions and customs. The Gujjars of Jammu and Kashmir are not an exception to this.

The Gujjars is a significant ethnic group of Jammu division occupying the plain, kandi land, the valley and side valleys of the lesser and the greater Himalayas. Gujjars are found in each and every district of Jammu and Kashmir. But the majority of them live in the Rajouri and Poonch districts of Jammu division. In the over all population of the state, Gujjars rank third in numerical strength. The Gujjars of the state can be divided into three sub groups,

The settled Gujjars,

The Homeless (transhumant) Bakarwals

Dhudia Gujjars.

On the basis of their socio-economic backwardness and mountainous terrain of their habitation, the Gujjars and Bakarwals of Jammu and Kashmir have been included in the category of scheduled tribes.

Despite ten five year plans and special Hill Area Development Programmes most of the Gujjars of Jammu and Kashmir in general and those engaged in transhumance in particular are struggling in abject poverty, living at a poor standard of nutrition having relatively short span of life. Their birth, death and infant mortality is high, their longevity is below 55 year, the literacy rate is low and the female literacy is one of the lowest in the country. The sex ratio is 850 females per 1000 males. In fact a significant proportion of their population is living below the poverty line. As per the growth, distribution and density of Gujjars at district level is concerned, prior to partition Jammu accounted about 37 per cent of Gujjars population. Since partition, due to migration to Pakistan the population decreased. In kathua and Jammu districts, particularly the kandi areas of both the districts are occupied by the Gujjars during winter and the plain areas are occupied by the Dhudia Gujjars in the tehsils of R.S Pura, Hira Nagar and important villages like Gujjar Nagar, Bathindi, Sanjwan, Chawadi, Harrian Chack.

The Udhampur district have the population and settlements of Gujjars in invariably scattered in hilly region of Gool Gulabgarh, Poni, Pharmari, Arnas and foothill areas of the villages of Ramnagar, chanani, Jhajjar Kotli, Mansar and Jindhrah villages. Doda district have sparsely populated Gujjar settlements that too only during summar season in Kishtwar and Bhadarwah tehsils and some settlements are found in Najwah, kasdan, Saradi, Chinta, Jahi, sunbain and Bhalesa.

The main density of population is found in the Rajouri and Poonch district, where more than 15 per cent of total population comprises of Gujjars settlement.

Table 1 Distribution of Gujjar Population in Poonch and Rajouri District

\begin{tabular}{|l|l|l|l|c|}
\hline S.NO. & District/Block & $\begin{array}{l}\text { Total } \\
\text { Population }\end{array}$ & $\begin{array}{l}\text { Gujjar } \\
\text { Population }\end{array}$ & Percentage of Gujjar Population to total population \\
\hline 1 & Poonch & 46273 & 25136 & 54.32 \\
\hline 2 & Mendhar & 78680 & 36410 & 46.30 \\
\hline 3 & Surankote & 92000 & 38737 & 42.10 \\
\hline 4 & Mandi & 47047 & 13802 & 29.33 \\
\hline 5 & Balakote & 21295 & 5521 & 25.90 \\
\hline 6 & Rajouri & 69126 & 31337 & 45.30 \\
\hline 7 & Darhal & 71168 & 29317 & 41.20 \\
\hline 8 & Budhal & 92826 & 48719 & 52.50 \\
\hline 9 & Manjakote & 55164 & 19504 & 36.30 \\
\hline 10 & Kalakote & 37370 & 12532 & 33.50 \\
\hline 11 & Nowshera & 50713 & 7518 & 14.80 \\
\hline 12 & Sunderbani & 43381 & 3001 & 6.90 \\
\hline
\end{tabular}

Source: Village Amenity Directory of District Rajouri and Poonch, 2002 
Particularly domination of Gujjars populations in Budhal, Kalakote, and Thanamandi, tehsils of Rajouri district and in Poonch district main Gujjars population is spread by permanent agricultural villages of tehsils Mendhar, Surankote and sparsely in tehsils Haveli. The important blocks of Gujjars settlement are Bafliaz, Samote, Balakote, Mendhar, Harni, Mankote blocks of district Poonch and Kalakote, koteranka, Dasal, Thanamandi, Manjakote, and Doongi blocks of Rajouri district.

The prominent settlement of Gujjars density are Fatehpur, Dodasan Bala, Plangarh, Mangota, Dara, Dhok, Painkhad, Gujjar Prori, Learadh, Jamola, Danna, Nadian, Kasbrari, Daramam, Seem Sumit and Dassal Gujran are the major settlements in Rajouri district. In district Poonch the main settlements with high density and population concentration are Bandi-chehian, Chandak, Lassana, Kassana,Sangala, Kalaban, Mandole, Hari,Mehrote, Seindara, Kasbrari, Narole. In these villages the density of population is more than 50per cent of the total population of the villages; otherwise every village and particularly the sample villages have 30per cent to 70per cent of Gujjar population.

Among the various components of population, sex composition, age composition and occupational composition, hold a prime place in population geography. The separate data for males and females are important for various types of planning and far the analysis of other demographic characteristics such as natality, mortality, migration, marital status and occupational characteristics. The balance of sex affects the social and economic relationships within a community. Since the two sex play partly contrasting and partly complementary roles in the economy and society, the study of sex composition assumes added significance for a scholar of population geography.

The study of sex ratio among the Gujjars had been a prominent portion of the research. While calculating from sample villages and the data of census after computing the sex ratio obtained is 856/100 among the Gujjar population. The low ratio of female is due to the early marriage in this community. Very less celebration takes place at winter location, mostly the Bakerwal sect celebrating their 'Nikah' at the summer location in Dhoks in a simple manner. Were no dowry is offered but dowry in the form of buffaloes among Gujjars and goats among Bakerwals is predominant in this community.

In the conservative male dominated society of Gujjars and Bakerwals, the health females is adversely affected by her own pious self-denial of life's comforts for the sake of her husband and children. Among the Gujjars the females are considered as economic and social liability by the parents while the males are credited as an asset to the family. The differences in the mortality rates of the males and females also give rise to differences in the sex-ratio. The females are more illiterate. All these factors together tell upon the health of the Gujjar-females and the result in relatively high female mortality. Consequently, the sex ratio among these tribals is highly skewed. In general, the sex among the scheduled tribes is high (982 females per 1000 males), but in the case of Gujjars and Bakerwals, it is only about 850 females per 1000 males. Their relative low sex ratio may be associated with relatively high female mortality rate among them.

The age composition of a population is determined by natality, mortality and mobility. These determinants of age structure are interdependent and any change in one of these may influence the other two and it is through these variables that the socio-economic conditions influence the age structure. It is the fertility that determines the proportion of population in different age categories. Mortality also affects the age composition of a population. The impact of migration upon age composition of Gujjars can be analyzed in a similar manner. Age composition of Gujjars and Bakerwals in the present study has been examined by categorizing the population in three age groups, i.e.(i) The young below 15 years, (ii) The adults - people falling in the age group of 15 to 59 years, and (iii) All those who have attained the age of 60 and above as old. This group is considered as an economic burden upon the adult age group as it is to provide with food, clothing and sufficient healthcare.

As far as age structure of Gujjar population is concerned, the composition is mainly found between 15 to 60 years age group. Being a working force the age grouping comprises upon 0-14 years which is about 30 to 35 per cent, 15 to 59 years which is about 60 to 65 per cent and above 60 years age the percentage is very low between 3.5 to 6.5 per cent. In fact, this population being a working class the middle age composition is predominant in the study region. Where as whole their structural phenomenon is almost similar to the other population living in the region.

The dependency ratio among the Gujjars of Rajouri and Poonch is significantly high. The dependency ratio has been computed by dividing the number of children plus old people by the number of adults (15-59 years age group) and multiplying it with one hundred. The dependency ratio is governed largely by the age structure of the populace.

So far as the manpower among the Gujjars of Rajouri and Poonch is concerned, a substantial proportion of their population is in the age group of 15 and 59. This age group has the propensity to participate in the economically gainful activities. It consists of both males and females. Economically not active population is that part of manpower, which is engaged in the activities like household duties in their own house or at the place of their relatives, retired personnel, intimates of institutions, students and those living on royalties, rents, dividends, pension etc. 
The concept of workers was introduced, for the first time, in India, 1961. According to the census of India, any person who main activity was participation in any economically productive worker either by his physical or mental activity was classified as worker (Census of India 1971, p. 169). Ever since the introduction of the concept in 1961, the definition of a worker in India has been changing from census to census.

The workforce in the Rajouri and Poonch districts constitute about 54per cent of the population. The females are actively involved in the outdoor economic activity like herding, tending of cattle, buffaloes, sheep and goats and the disposal of milk and milk products in the neighboring towns and periodic markets. There are however, regional variations in the workforce at the block and village levels.

The Gujjars of the region under study are largely dependent on primary economic activity herding (cattle, buffalo, sheep and goats keeping), cultivation of crops, collection of wood and forest products, etc. Less than 10per cent of the workforce is in the tertiary sector, while the secondary sector engages less than 2 per cent of the Gujjars workforce. Thus nearly 90per cent of the workforce is dependent on agriculture (domestication of animals and cultivation of crops) and forestry. Interestingly enough, the incidence female participation in outdoor economic activity is very high.

So far as minimum level of literacy skill is concerned the districts of Rajouri and Poonch are amongst the low literacy districts of the country. The literacy rate of Gujjars and Bakerwals is below 35per cent, while that of the females is below 10per cent. The lowest literacy rate is found among the Bakerwals who practice transhumance and oscillate between the alpine pasture (in summer season) to winter pastures in the plains and Kandi lands of the Siwalik. The strikingly low literacy rates among the females in the less developed part of the Jammu Division are the product of a variety of historical, social, economic and political factors. The prejudices against the females education in the male dominant society of Gujjars, prejudice against female mobility, the low status granted to the females, lack of educational institutions for the females are the main factors responsible for female illiteracy. Moreover, female literacy has a positive correlation with the status of women in the society. Among the Gujjars early marriage is prevalent. Thus, even those female children who join school at an early age have a tendency to drop out in the event of their marriage. In addition to these, there is a strong feeling among the parents that ultimately the female child has to live with the spouse; therefore, an investment on her education was uncalled for, particularly when parents cannot afford it.

Table 2

Literacy rate of Gujjars in Rajouri and Poonch districts (2002-03)

\begin{tabular}{|l|l|l|}
\hline S.No. & Name of the Blocks & $\begin{array}{l}\text { Percent } \\
\text { literate }\end{array}$ \\
\hline 1 & Poonch & 8.2 \\
\hline 2 & Mandi & 3.3 \\
\hline 3 & Balakote & 2.7 \\
\hline 4 & Mendhar & 6.3 \\
\hline 5 & Surankote & 5.5 \\
\hline 6 & Rajouri & 14.1 \\
\hline 7 & Manjakote & 2.9 \\
\hline 8 & Nowshera & 5.3 \\
\hline 9 & Kalakote & 3.7 \\
\hline 10 & Budhal & 4.1 \\
\hline 11 & Darhal Malikan & 4.2 \\
\hline 12 & Sunderbani & 2.7 \\
\hline
\end{tabular}

Source: Based on Sample survey (2002-2003)

In the districts of Rajouri and Poonch, the Gujjars appalling poverty and migratory character of the seasonal migrants are the factors responsible for wide disparity in the male-female literacy rates. The Gujjars who are settled in agriculture and having a good literacy rate such those like transhumant Bakerwals and Dhudia Gujjars are having low profile of literacy. Particularly, there is a small gap in male and female literacy rate. Female literacy is very low at district, Blocks and village levels, as revealed from the study sample villager. Still the Gujjars particularly the oscillatory section is reluctant to send the female children to school which has results the ratio of female literacy very low.

Seasonal migration is one of the important characteristics of a significant proportion of the Gujjars of the Rajouri and Poonch districts. Pastoral nomadism of the transhumance type, as practiced in the study area at present is the manifestation of the interaction between the distinctive environmental conditions of the region and the changing socio-economic context from time to time and from place to place. A recurrent theme in studies relating the transhumance has been the use of environmental models to explain the origin, development and the characteristics of this distinctive mode of living. Thus the classical explanatory models explicitly or implicitly 
associate the practice with land and to specific environmental characteristics. Generally it is the theorized that exigencies of a long, dry summers in the lowlands or the foothills which make pastures scarce, necessitates an upward migration of the stock to high altitude grasslands for summer pasturing. The resultant regular oscillatory movement of men and flock is seen as a socio-economic response to this specific feature of the environment, epitomizing the symbolic interdependence between man, animal and environment.

In the spatial context of the study area the environmental explanation seeks to depict the practice of transhumance in terms of the seasonal distribution of pastures which calls for a to and fro movement of men and flock between winter pasture in the Pir-Panjal foot-hills and the summer pastures in the Alpine meadows of outer Himalayas as a component of the symbiotic relationships. The socio-economic organization of the people is not viewed as a specific grazier.

The environmental explanation of seasonal migration is however, over simplifications of a complex man-environmental interface. A review of current works on the subject clearly demonstrates that transhumance is neither ubiquitously present nor continuously practiced in similar environments. The inadequacy of the environmental explanation of transhumance is now clearly reorganized and the emphasis in recent works on the subject has clearly shifted from the assumed environmental complementary to focus more clearly and critically on historically and cultural factors which can provide more reasonable explanation. It is also now being opened that transhumance represents not the inevitable consequence of particular pre-determined conditions, but rather a rational means of assessing and utilizing physical and human resources more efficiently.

It is more important to examine contingent conditions, rather than fixed environment. In accordance with this shifting emphasis recent studies of transhumance tend be concentrate more on specific characteristics of the practice such as pasturing techniques rather than viewing no practice in general environmental terms.

However, a more correct methodological position would be not emphasis too much either the environment or the culture. A distinction can usefully be made between the base structure of transhumance, which may reflect in a general way the role of ecological factors between regions and variations of practice reflecting social and economic society dichotomy, may well represent a more realistic interpretation of transhumance.

The environmental framework within which transhumance take place in the study region is characterized by great contrast in summer conditions in the foothill zone of the Jammu region and the highlands of the outer Himalayas. The transhumance economy of necessity seasonally oscillates between these two points. This is an environmental imperative. There is an utter contrast in summer conditions between the foothills and the high Himalayas. During the summer months the foothill pastures whether away under intense heat. There is not enough pasturage left the pastoral nomadies. Therefore, have no option but to make a move towards new verdant woods and pastures green. Thus for centuries seasonal deficiencies of fodder or water in parched lowlands, thereby establishing the annual rhythm of transhumance between lowlands and highlands. There is, therefore, little doubt the antiquity of the practice in Jammu region. It can safely be presumed that the local Gujjars and Bakerwals have been transhuming ever since they colonized the region. Literacy evidences indicate the presence of the practice in the region much before the Mughal period. The practices remained important until recent times.

Centuries of transhumance resulted in the establishment of a symbiotic relationship between the GujjarBakerwal community, their stock and their environment. A set of social norms and familial and group relationship evolved in order to harmonize the culture economy and society with the physical attributes of the environment. It is, therefore, not surprising that arose in the region a distinct ethos and a general Genere-de-vie. The persistence of this practice for centuries across a range of ecosystem, ranging from the foothills, the middle mountains, the Kashmir valley and high altitude alpine pastures, is a testimony to the resilience system and adaptability of transhumance as a viable as a socio-economic system. The environmental origin of transhumance in the region and economy of the people was an ideal response the ecological factors.

However, in recent years a number of distinct changes in the geography of transhumance in the study region have been observed. The social and economic context has started exerting a great influence on the nature and function of transhumance. The environmental influences cannot by themselves account for the development and changing character of the pastoral practice. The physical environment has not changed the locational complimentarity of the summer and winter pastures has not altered a bit. Winter pasturing remains confined to the foothills while the chief summer pastures continue to be at higher elevation. Transhumants herds still move to and fro between these pasture zones. The major changes that are now taking place relate to the basic structure and viability of this form of pastoral nomadism distinct areas of Gujjar sedentary concentration have emerged, in the foothill zone and with side valleys. This development reflects a general decline of transhumance even though the environmental framework remains the same.

It has also been observed in the state of Jammu and Kashmir affluence and transhumance are inversely proportional only the poorest section of the community is still truly transhumant while the effluents have by a large served their link with the migratory mode of living and have settled down in agriculture, trade or some 
other economic activities. It can be said that, at least in the context of the study area, transhumance as of now, is not environmentally ordained but is rather the function of the stage of development and its concomitant economic capacity to switch over a new socio-economic order. This is born out by the fact that in the Jammu and Kashmir the affluent section s of the Gujjar- Bakarwals communities have more or less completely discarded pastoral nomadism as a way of life and have permanently settled down. It is only the poorest of the Bakarwals who still adhere to transhumance. Indeed a vertical mobility is clearly discernable in the social structure, dependent upon the degree of sedentrization.

In brief, there is a conspicuous shift from transhumance to agricultural and non-agricultural sectors among the Gujjars and Bakarwals. The process of sedentrization of the seasonally migrating population of Gujjars may get accelerated if infrastructure for the development of secondary and tertiary sectors can be developed. The small and marginal Bakarwals as well as the very large of them having higher social st5atus are getting eliminated from transhumance to sedentrization in the side valleys in the Himalayas. The sustainability of sedentrization depends on the development of strong infrastructure in the form of roads, electricity, irrigation and industrial sector. Sooner a strong infrastructure provided the better for the upliftment and socio-economic transformation of the Gujjars and Bakarwals of the western Himalayas in general and that of the Rajouri and Poonch districts in particular.

\section{References}

[1]. Bandarker, D.R. (1905): Wondering culture of Central Asia. J. Royal Asiatic society, p.1

[2]. Bhardwaj, A.N, (1994). History and culture of Himalayan Gujjars, jay kay publication, Jammu, pp.1-16

[3]. Bhat. A.R (2005), Human resource and socio-economic development in Kashmir valley: A Geographical interpretation, Dilpreet publ. house, New Delhi, pp. 42-51

[4]. Census of India (1961): Gojjari Speaking Population in Jammu and Kashmir, Vol. XXIV. Part II, p.241.

[5]. Hussain, M (2000), systematic geography of Jammu and Kashmir, Rawat Publication. New Delhi, pp.121-123.

[6]. Kanedy, j. (1923): Nomadic Gujjars of Hindukush. J. Gr. Brit. Asiatic society: pp. 153-154

[7]. Khatana, R.P. (1986). Gujjar Bakarwal Transhumance in Jammu and Kashmir, Arina publ. house, New Delhi, pp.86-115.

[8]. Lidhoo, M.L (1985). Gujjars: Their Attitude towards Social Change, Education and Modernization, pp.2-15

[9]. Manku, D.S (1986). The Gujjar Settlements: A Case Study in Ethnic Geography, Inter-India, Publication, New Delhi. Pp.29-30

[10]. Mir, A.M. (2002): Geography of Jammu: A Regional Analysis. Dilpreet Publ. house, New Delhi, pp 40-41

[11]. Munshi K. M (1954). Ret Raj Gujjar Desh. The Glory, New Delhi. Pp.6-9

[12]. Zutshi, B. (1998). Population distribution of Jammu and Kashmir: A Regional Analysis in Geography of Jammu and Kashmir, New Delhi, pp.199-213. 